\title{
Prevention of chemotherapy-induced alopecia: experience of a cancer center in Romania
}

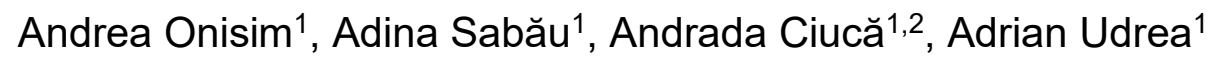 \\ ${ }^{1}$ Medisprof Cancer Center, Cluj-Napoca, Romania \\ ${ }^{2}$ Babes-Bolyai University, Department of Psychology, Cluj-Napoca, Romania \\ Corresponding author: Andrea Onisim e-mail: andreaonisim@medisprof.ro
}

\section{Abstract}

Background: Chemotherapy still represents the backbone of systemic treatment for many solid tumors. Alopecia remains one of its most distressing side effects. Scalp cooling is an effective strategy to reduce the risk of alopecia induced by chemotherapy, therefore this study aimed to assess the efficiency of DigniCap scalp-cooling system in our center.

Material\&Methods: 113 patients diagnosed with solid tumors, who underwent chemotherapy using the DigniCap scalp-cooling system in our center between January 2018 - December 2020, were included in the study. Alopecia was evaluated by the physician according to Dean's scale at baseline and after the last cycle of chemotherapy. The primary endpoint was to evaluate scalp-cooling efficiency defined by a Dean's scale score of 0-2 (hair loss $\leq 50 \%)$. Results: $78(72.3 \%)$ out of the 113 patients who used scalp cooling presented hair loss of $50 \%$ or less, the majority were females $(91.2 \%)$ and had a diagnosis of breast cancer $(49.6 \%)$ or gynecological cancer (27.4\%). The mean number of chemotherapy cycles using DigniCap was 4.82 cycles. Hair loss greater than $50 \%$ of the scalp surface was observed in $27.7 \%$ of patients, the highest grade of alopecia being observed for anthracycline-based chemotherapy. No serious adverse events were reported.

Conclusion: The results of this study support the evidence existing so far in the literature. Among patients who underwent chemotherapy for various cancer types, the DigniCap System significantly prevented hair loss and we consider that it should be integrated in the supportive care of patients.

Keywords: alopecia, chemotherapy, DigniCap, patients, scalp-cooling 


\section{Background}

According to the World Health Organization (WHO), cancer is the second leading cause of death globally. Female breast cancer surpassed the incidence of lung cancer, followed by colorectal, prostate and stomach cancer [1]. In spite of novel therapies, chemotherapy still remains the backbone of systemic therapy for a wide range of cancers, either in curative neoladjuvant or metastatic setting. Some of these regimens may induce alopecia as a side effect, causing a lot of distress for the patients, especially for women. The physical appearance is perceived to be the main source of psychological discomfort, even though other toxicities are more serious complications. In order to reduce the risk of chemotherapy induced alopecia, scalpcooling systems evolved over the last decades with automated devices gaining FDA approval after several clinical trials, proving their safety and efficacy and thus giving hope to cancer patients [2,3]. Scalpcooling systems are used in many countries to prevent alopecia, however they are not reimbursed in all countries, including Romania. In this context, with most of the data coming from Western countries, this study aimed to assess our own experience and results with DigniCap scalp-cooling system.

\section{Material \& Methods}

Study design and patients

We conducted a prospective, crosssectional study, including 113 patients who underwent chemotherapy using DigniCap scalp-cooling system, between January 2018 - December 2020, at Medisprof Cancer Center, Cluj-Napoca, Romania. Patients were considered eligible if they were treated with chemotherapy agents that induce alopecia in the neoadjuvant, adjuvant or metastatic setting. Exclusion criteria were ECOG performance status greater than 1, hematologic tumors, history of brain metastases treated with radiotherapy, other causes of alopecia, as well as cold agglutinin disease, cryoglobulinemia or cold sensitivity. All patients who agreed to participate in the study signed an informed consent. Clinical characteristics of patients, including age, diagnosis, stage and treatment setting, chemotherapy regimens and number of cycles, were collected by reviewing the patient electronic file.

The DigniCap scalp-cooling system was used on the day of chemotherapy administration, from cycle 1 until the end of chemotherapy or earlier in case of unsatisfactory results or side effects. The device consists of a suitable size silicone cap in which a glycol-based liquid coolant, is recirculated through two channels connected by sensors to a computerized cooling and control unit. The system is initiated aprox. 30 min before treatment, the cooling is gradual until the optimal temperature of $3-5^{\circ} \mathrm{C}$, which is maintained throughout chemotherapy and for $90 \mathrm{~min}$ to $120 \mathrm{~min}$ afterwards, depending on the chemotherapeutic agent and the dose. Blood vessels constriction takes place in the scalp, as well as a reduction of the cell 
division in the hair follicles, making them less vulnerable to chemotherapy.

\section{Endpoints}

The primary endpoint of our study was to assess the efficiency of DigniCap scalpcooling system in the prevention of hair loss induced by chemotherapy in patients with solid tumor. The degree of alopecia was evaluated by the clinician using Dean's scale [4]: grade 1 (<25\% hair loss), grade 2 (25\%$50 \%$ hair loss), grade 3 (50\%-75\% hair loss), and grade 4 (>75\% hair loss). Clinical assesements of patients regarding alopecia were performed at baseline and 3-4 weeks after the last cycle of chemotherapy and DigniCap. Scalp-cooling was considered effective when less than $50 \%$ hair loss recorded (grade 0-2) [4].

As a secondary endpoint we aimed to evaluate patients' perception of the device, potential adverse events and quality of life aspects, by filling a brief questionnaire adressing these issues experienced during chemotherapy. 76 of the 113 patients included in the study completed this questionnaire. Concern about potential adverse events related to chemotherapy (such as fatigue, nausea, hair loss, skin and nails changes, renal and hepatic impairment) were scored from 1 (a little worried) to 5 (very worried). Quality of life topics (such as the importance of body image, the fear of hair loss, the impact of alopecia on personal life, tendency to depression, the need of psychological support and emotional support of loved ones) were adressed through 10 questions. Each topic received a score from
1 to 4 (1- no impact; 2- a little bit; 3- quite a lot; 4-very much).

\section{Statistical analysis}

In the present research, the statistical analysis was performed with the IBM SPSS v.20. Descriptive statistics were used to summarize the clinical characteristics. The quantitative variables were summarized as mean and standard deviation, while qualitative variables were expressed as absolute and relative frequencies (\%). In order to investigate potential differences between various quantitative and qualitative nominal variables independent-sample t-test and one-way ANOVA test were used. A significance level of 0.05 was used for all twosided statistical tests, and a test result was considered significant when $p$ was lower than 0.05 .

\section{Results}

A total of 113 patients who underwent alopecia inducing chemotherapy, participated in the study, of which 103 women and 10 men. The mean age of treated patients was 54 years (range, 26-77 years). Detailed characteristics of the participants are presented in Table 1. The majority of patients who used DigniCap were those with breast cancer (49.6\%), followed by gynecological (27.4\%), gastrointestinal $(9.7 \%)$ and lung cancer $(7.1 \%)$. Patients with prostate and bladder cancer $(4.4 \%)$ or other sites such as head and neck or melanoma $(1.8 \%)$, who received alopecia inducing drugs, were less likely to opt for scalp cooling. Most patients $(56.6 \%)$ received 
chemotherapy with curative intent in a underwent treatment in a metastatic setting. neoadjuvant or adjuvant setting, while $43.4 \%$

Table 1. Characteristics of patients in the study group

\begin{tabular}{|c|c|c|c|}
\hline & & $\mathbf{N}$ & $\%$ \\
\hline \multirow[t]{2}{*}{ Sex } & Male & 10 & 8.8 \\
\hline & Female & 103 & 91.2 \\
\hline \multirow[t]{7}{*}{ Diagnosis } & Breast & 56 & 49.6 \\
\hline & Gynecological & 31 & 27.4 \\
\hline & Colorectal & 6 & 5.3 \\
\hline & Digestive & 5 & 4.4 \\
\hline & Genitourinary & 5 & 4.4 \\
\hline & Pulmonary & 8 & 7.1 \\
\hline & Others & 2 & 1.8 \\
\hline \multirow[t]{4}{*}{ Chemotherapy Type } & Anthracycline-based & 32 & 28.3 \\
\hline & Taxane-based & 57 & 50.4 \\
\hline & Anthracycline + Taxane & 17 & 15.0 \\
\hline & Other agents & 7 & 6.2 \\
\hline \multirow[t]{3}{*}{ Treatment Setting } & Neo-adjuvant & 45 & 39.8 \\
\hline & Adjuvant & 19 & 16.8 \\
\hline & Metastatic & 49 & 43.4 \\
\hline Alopecia degree & 0 & 14 & 13.0 \\
\hline \multirow[t]{5}{*}{ (Dean scale) } & $1(0-25 \%)$ & 38 & 35.2 \\
\hline & $2(25-50 \%)$ & 26 & 24.1 \\
\hline & $3(50-75 \%)$ & 19 & 17.6 \\
\hline & $4(>75 \%)$ & 11 & 10.2 \\
\hline & & Mean & SD \\
\hline Age in years & & 54.19 & 11.06 \\
\hline Total cycles & & 5.97 & 2.56 \\
\hline Total cycles with & & 4.82 & 3.01 \\
\hline Dignicap & & & \\
\hline
\end{tabular}


The most common chemotherapy regimens administered were taxane-based $(50.4 \%)$ or anthracycline-based (28.3\%). However, $15 \%$ of patients who used DigniCap received both anthracyclines and taxanes. The chemotherapy regimens used are illustrated in Fig. 1. In several cases,
Bevacizumab was associated to Paclitaxel/Carboplatin or Pertuzumab and Trastuzumab to taxane-based chemotherapy. The mean for the total number of chemotherapy cycles administered has been 5.97 , whereas the mean for the number of cycles with DigniCap has been 4.82 .

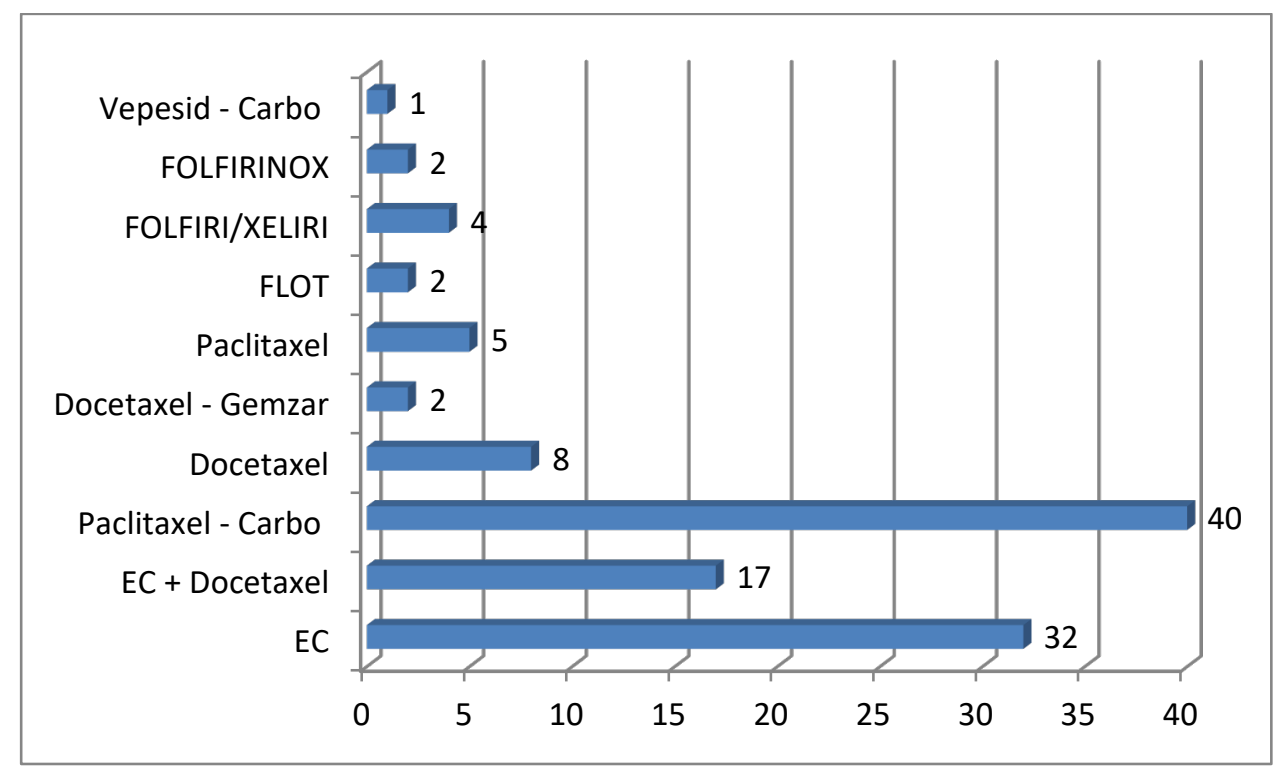

Fig. 1 Patient distribution according to chemotherapy regimens

Of the 113 patients who used scalp cooling, 78 (72.3\%) demonstrated hair loss of $50 \%$ or less (Dean score of $0-2$ ). The best results, with less than $25 \%$ hair loss were recorded in $48.2 \%$ of patients, whereas severe alopecia (Dean score 3-4) despite using DigniCap was objectified in $27.7 \%$ of cases. Seventy-six of the 113 patients included in the study completed our questionnaire adressing potential adverse events related to chemotherapy and quality of life aspects. The mean scores for each item are shown in Table 2. Scores were similar for fatigue, nausea, skin and nail changes, renal and liver dysfunction, however the highest score (Mean=4.08, $\mathrm{SD}=1.44$ ) has been registered for alopecia. For $49(64.4 \%)$ of the 76 patients analyzed, hair loss has been a major concern related to chemotherapy.

Quality of life analyzed items showed a significant impact, translated into the highest scores for: the importance of body image, the fear of hair loss and the impact of alopecia on personal life. The mean score for depression revealed a tendency to depression. The need of psychological support and emotional support of loved ones 
were also highly scored. However, selfreported hair loss and side effects encountered with DigniCap received the lowest scores. Side effects related to
DigniCap were chills reported by 21 patients, headaches in 6 cases and heavy head/dizziness in 4 patients.

Table 2. Adverse Events Concerns and Quality of Life

\begin{tabular}{llc} 
Scores & & \\
\hline Adverse Events Concerns & Mean & SD \\
\cline { 2 - 3 } Fatigue & 2.83 & 1.58 \\
Nausea & 2.66 & 1.57 \\
Hair loss & 4.08 & 1.44 \\
Skin and nails changes & 2.57 & 1.50 \\
Liver and renal dysfunction & 2.72 & 1.59 \\
\hline Quality of Life & & \\
Body image & 3.27 & 0.84 \\
Risk of hair loss & 3.36 & 0.82 \\
Hope with DigniCap & 3.36 & 0.86 \\
Side effects with DigniCap & 1.86 & 0.84 \\
Self-reported hair loss & 1.49 & 0.71 \\
Depression & 2.86 & 0.98 \\
Impact of alopecia on personal life & 3.78 & 0.55 \\
Emotional support of loved ones & 2.74 & 1.02 \\
Psychological support & 3.44 & 0.85 \\
& & \\
\hline Type of side effects with DigniCap & $\mathbf{N}$ & $\%$ \\
Headache & 6 & 7.89 \\
Feeling cold & 21 & 27.63 \\
Dizziness & 4 & 5.26 \\
Nausea & 2 & 2.63 \\
\hline
\end{tabular}

An independent-sample $t$ test was performed in order to analyze differences between age groups in terms of alopecia degree. There was no statistically significant difference observed between groups, $t(104)=0.88, p=0.379$. Descriptive statistics are shown in Table 3, the mean score for alopecia was 1.82 , in the subgroup under 65 years, respectively 1.59 in the group over 65 years. 
Table 3. Alopecia according to age groups

\begin{tabular}{|l|r|r|r|}
\hline Age groups & $\mathbf{N}$ & Mean & Std. Deviation \\
\hline$<65$ years & 85 & 1.82 & 1.206 \\
\hline$\geq 65$ years & 28 & 1.59 & 1.047 \\
\hline
\end{tabular}

In order to explore the differences regarding alopecia according to gender, we used an independent-sample $t$ test that showed statistically significant differences between males and females, $\mathrm{t}(104)=3.73$, $p<0.001$. The mean score for alopecia was 1.87, $S D=1.12$, in the female subgroup, while in males it was $0.44, S D=0.72$. The difference is illustrated in Fig. 2.

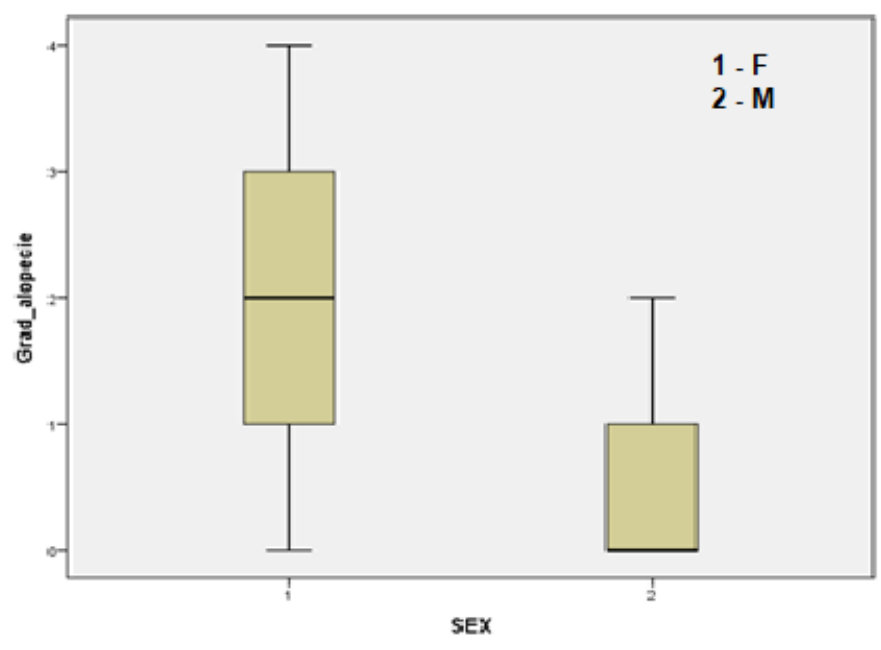

Fig. 2 Alopecia according to gender

Further on, we aimed to analyze whether there are differences between the degree of alopecia and the site of primary tumor. Oneway ANOVA was performed and there were statistically significant differences observed between groups, $F(6,101)=8.29$, $\mathrm{p}<0.001$.
Descriptive statistics are shown in Table 4, the highest score for alopecia was observed for breast cancer patients, whereas the lowest score was seen in prostate cancer patients that used DigniCap and received chemotherapy with docetaxel.

Table 4. Alopecia according to cancer site

\begin{tabular}{|l|r|r|r|}
\hline Cancer site & $\mathbf{N}$ & Mean & Std. Deviation \\
\hline Breast & 56 & 2.33 & 1.055 \\
\hline Gynecologic & 31 & 1.31 & 0.891 \\
\hline
\end{tabular}




\begin{tabular}{|l|r|r|r|}
\hline Colorectal & 6 & 0.33 & 0.516 \\
\hline Other digestive & 5 & 1.20 & 0.837 \\
\hline Genitourinary & 5 & 0.25 & 0.500 \\
\hline Lung & 8 & 1.71 & 1.254 \\
\hline Other & 2 & 1.00 & $<0.001$ \\
\hline Total & 113 & 1.75 & 1.161 \\
\hline
\end{tabular}

We then proceeded to a post hoc analysis using the Tukey HSD (Honestly Significant Difference) test. These comparisons, having alopecia as a dependent variable, indicated significant differences between breast cancer and gynecological cancers (Mean=1.017, SD= $0.224, \quad p<0.001)$, breast cancer and colorectal cancer (Mean=1.994, SD=0.421, $p<0.001)$, breast cancer and genitourinary cancers (Mean=2.077, SD=0.507, $p=0.002$ ).
An independent-sample t test was further performed in order to analyze the differences in terms of alopecia degree according to treatment setting. There was a statistically significant difference observed between groups, $t(104)=4.82, \quad p<0.001$. Descriptive statistics shown in Table 5, emphasized for neoadjuvant or adjuvant chemotherapy a mean for alopecia score of 2.17, $S D=1.144$, whereas for metastatic setting the mean for alopecia score was $1.16, \mathrm{SD}=0.924$.

Table 5. Alopecia according to treatment setting

\begin{tabular}{|l|r|r|r|}
\hline Treatment setting & $\mathbf{N}$ & Mean & Std. Deviation \\
\hline Adjuvant/neoadjuvant & 64 & 2.17 & 1.144 \\
\hline Metastatic & 49 & 1.16 & 0.924 \\
\hline
\end{tabular}

Eventually, a one-way ANOVA was performed in order to analyze differences in terms of alopecia degree according to chemotherapy regimens administered using DigniCap. The differences observed between groups were statistically significant, $F(3,102)=34.77, \quad p<0.001, \quad$ and are illustrated in Fig. 3. The highest mean for alopecia degree was observed for anthracycline-based chemotherapy (Mean=3.03, SD=0.795), while for taxane-based regimens the mean for alopecia scores was lower (Mean=1.35, $S D=1.41$ ). However, for patients who used Dignicap with both anthracyclines and taxanes the mean for alopecia was also lower (Mean=1.41, SD=0.618). Regimens containing other agents such as Irinotecan or Etoposide were associated with the lowest mean of alopecia scores (Mean=0.29, $\mathrm{SD}=0.488)$. 


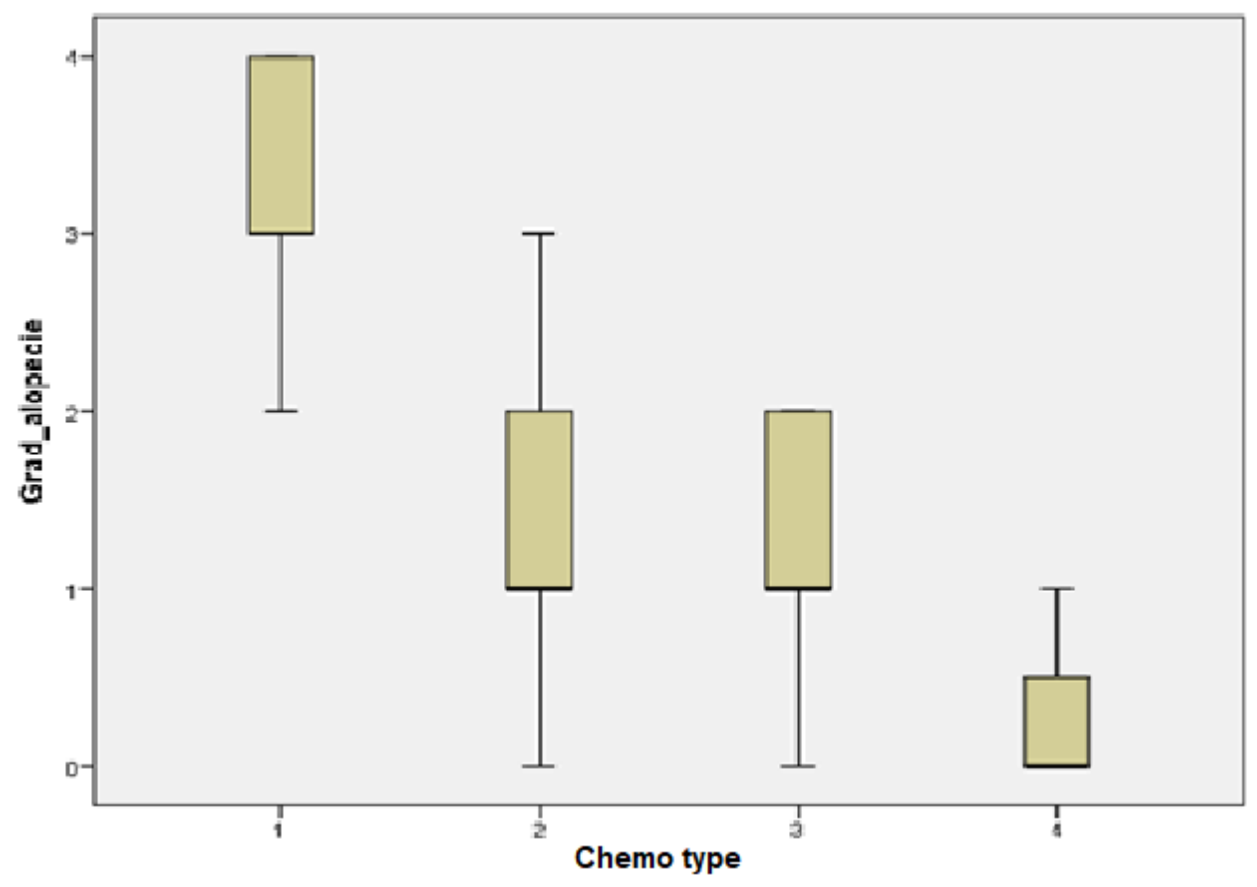

Fig. 3 Alopecia according to chemotherapy type

(Chemo type - 1: anthracycline-based; 2: taxane-based; 3: anthracycline+taxane; 4: other agents. Grade of alopecia - 0: 0\%; 1: 0-25\%; 2: 25-50\%; 3: 50-75\%; 4: >75\%).

\section{Discussion}

In this prospective study we demonstrated that the scalp-cooling system DigniCap was able to prevent alopecia (less than $50 \%$ hair loss) in $72.3 \%$ of patients with solid tumors who received chemotherapy regimens that induce alopecia. In all participants, $48.2 \%$ had less than $25 \%$ hair loss. Alopecia greater than $50 \%$ of the scalp surface was observed in only $27.7 \%$ of patients who used DigniCap, the majority $(80 \%)$ due to anthracyclines. Data reported by previous clinical studies focused mostly on women undergoing chemotherapy for breast cancer $[5,6,7]$. In a recent multicentre randomized clinical trial including women with early-stage breast cancer undergoing taxane and/or anthracycline-based chemotherapy, Nangia et al. confirmed efficacy of scalp-cooling in $50.5 \%$ of women [8]. Another prospective study conducted by Rugo et al., reported success of Dignicap in preventing alopecia in $66.3 \%$ of patients with early breast cancer [9].

Almost half of the patients included in this study had breast cancer diagnosis. However, our main goal was to evaluate efficacy of DigniCap in various solid tumors and to our knowledge this is the first data reported from an Eastern European country. Recent systematic reviews and metaanalyses of randomized controlled trials including different scalp cooling techniques, from non-automated to digitally controlled systems, sustained the positive effect of 
scalp cooling in reducing the risk of alopecia in patients with solid tumors [10,11]. Consequently, in 2017 automated scalp-cooling devices have been approved by the Food and Drug Administration (FDA) [12]. Moreover, European Society for Medical Oncology (ESMO) has updated in 2020 the guidelines for dermatological toxicities related to anticancer agents, including scalp cooling as a category IIB recommendation for prevention of chemotherapy induced alopecia [13].

When we analyzed the results according to different characteristics, we observed that age did not influence the outcome regarding the risk for alopecia. However, a difference was found between genders. The female subgroup seemed to be at higher risk, yet in order to draw conclusions a more even distribution of the males and females and regimens in the study would be required. This might also be explained by the highest rates of alopecia being found in the breast cancer group, followed by gynecological cancer group, which include only female patients. Therefore, anthracyclines remain the main hair-loss inducing chemotherapy. There were patients who received both anthracyclines and taxanes and the mean score for alopecia was lower compared to those receiving anthracyclines only. This could be explained by the fact that patients who experienced significant hair-loss during anhracycline chemotherapy were less likely to use the scalp cooling system later on. On the other hand, if the degree of alopecia was significantly low in the anthracycline setting, the risk for further alopecia was not increased when patients continued with taxanes.

However, considering that we evaluated patients with various cancer sites, the main chemotherapy regimens used were taxanebased and the overall analysis showed that taxanes are at lower risk for inducing alopecia. Moreover, since breast cancer patients were the majority and anthracyclines are more commonly used in neoadjuvant or adjuvant setting, the mean score for alopecia was higher in the curative treatment subgroup compared to the metastatic setting. The results of a recent prospective study showed that alopecia has been most successfully prevented in patients with early breast cancer and ovarian cancer treated with taxane +/carboplatin regimens [14].

Patients' concerns about potential adverse events were similar, whether we were talking about fatigue, nausea, nail and skin changes or renal and liver impairments related to chemotherapy. The fact that alopecia received the highest score proves why using scalp cooling systems should be considered for every patient at risk. Quality of life questionnaires should be handed to all patients. In our study, items related to the physical appearance were rated higher. Also, the hope that Dignicap system would prove itself to be efficient was one of the main expectations. However, regardless of patients' concerns, psychological support should be provided. Of the 76 patients who completed the questionnaire, the reported side effects related to the 
scalp cooling system were chills, followed by dizziness and headaches, however most of them were mild. Similar side effects were reported in the literature $[15,16]$. Regarding quality of life, a recently published systematic review showed that results are still inconsistent [17]. However, the majority of the patients who experienced effective scalp cooling reported better well-being, whereas the impact on QoL seems to be worse when scalp-cooling proved to be ineffective $[6,18,19]$.

A limitation of our study is the lack of a sur control arm and randomization, in order to evaluate more accurately the differences regarding the results and hair recovery between those who used DigniCap and those who chose not to try it. Hair volume and length should also be considered for more accurate information regarding the benefit of scalp cooling each institution, given the additional costs systems. Moreover, longer follow-up related to the need of dedicated personnel is needed to assess long-term safety, and longer duration of the treatment and especially the risk of scalp metastases. The time spent in clinic by the patient [6].

\section{Abbreviations}

ECOG - Eastern Cooperative Oncology Group

ESMO - European Society for Medical Oncology

FDA - Food and Drug Administration

HSD - Honestly Significant Difference

WHO - World Health Organization

\section{Statements}

\section{Authors' contributions:}

Andrea Onisim: study design, research, wrote the paper, conceived the analysis

Adina Sabau: study design, research, wrote the paper, conceived the analysis

Andrada Ciuca: statistical analysis

Adrian Udrea: study design, review of the manuscript 
Consent for publication: As the corresponding author, I confirm that the manuscript has been read and approved for submission by all co-authors.

Conflict of interest: All authors declare having no competing interests associated With this publication.

Funding Sources: This paper was supported by the project POC-Al-Al.1.1-A-2015- \&quot; Research laboratory on personalized therapy in oncology\&quot;, a project co-funded through the Operational Program Competitiveness 2014-2020.

Statement of Ethics: The accompanying manuscript does not contain experimental studies, Dignicap system has been approved by FDA and ESMO.

Ethical Approval: The treatment strategy/study protocol was approved by a local ethics committee.

\section{References}

1. Sung H, Ferlay J, Siegel RL et al. Global cancer statistics 2020: GLOBOCAN estimates of incidence and mortality worldwide for 36 cancers in 185 countries. CA Cancer J Clin 2021 Feb 4. doi: 10.3322/caac.21660. Online ahead of print.

2. Ross M, Fischer-Cartlidge E. Scalp cooling: a literature reviewof efficacy, safety, and tolerability for chemotherapy-inducedalopecia. Clin J Oncol Nurs 2017;21:226-33.

3. Fischer-Cartlidge E, Ross M, Hernández K, Featherstone A, Haase C. Scalp Cooling: Implementation of a Program at a Multisite Organization. Clin J Oncol Nurs 2018;22(5):534-541.

4. Smetanay $K$, Junio $P$, Manuel Feißt $M$ et al. COOLHAIR: a prospective randomized trial to investigate the efficacy and tolerability of scalp cooling in patients undergoing (neo)adjuvant chemotherapy for early breast cancer. Breast Cancer Res Treat 2019;173(1):135-143.

5. Villarreal-Garza C, Mesa-Chavez F, Garza-Ledezma MRA et al. Impact of chemotherapy regimen and sequence on the effectiveness of scalp cooling for alopecia prevention. Breast Cancer Res Treat 2021;185(2):453458.

6. Munzone E, Bagnardi V, Campennì G et al. Preventing chemotherapy-induced alopecia: a prospective clinical trial on the efficacy and safety of a scalp-cooling system in early breast cancer patients treated with anthracyclines. Br J Cancer 2019;121(4):325-331.

7. Giarratano T, Frezzini S, Zanocco M et al. Use of scalp cooling device to prevent alopecia for early breast cancer patients receiving chemotherapy: A prospective study. Breast J 2020;26(7):1296-1301.

8. Nangia J, Wang T, Osborne $C$ et al. Effect of a scalp cooling device on alopecia in women undergoing chemotherapy for breast cancer: The SCALP randomized clinical trial. JAMA 2017;317:596-605. 
9. Rugo HS, Klein P, Melin SA et al. Association between use of a scalp cooling device and alopecia after chemotherapy for breast cancer. JAMA 2017;317:606-614.

10. Rugo HS, Voigt J. Scalp hypothermia for preventing alope-cia during chemotherapy. a systematic review and meta-analysis of randomized controlled trials. Clin Breast Cancer 2018;18:19-28.

11. Shah VV, Wikramanayake TC, DelCanto GM, et al. Scalp hypothermia as a preventative measure for chemotherapy-induced alopecia: a review of controlled clinical trials. J Eur Acad Dermatology Venereol 2018;32:720-734.

12. Mustoe MM, Lee CM, Melisko ME, Esserman LJ, Hope S, Rugo HS. The DigniCap Scalp Cooling System and its use in the treatment of chemotherapy-induced alopecia. Future Oncol 2018;14(24):2461-2469.

13. Lacouture ME, Sibaud V, Gerber PA et al. Prevention and management of dermatological toxicities related to anticancer agents: ESMO Clinical Practice Guidelines. Ann Oncol 2021; 32(2):157-170.

14. Papadopoulos L, Chua S, Goss G et al. Prevention of chemotherapy-induced alopecia: A prospective study in breast and ovarian cancer patients [abstract]. Cancer Res 2021;81(4 Suppl):Abstract nr PS13-36.

15. Pedersini R, Fornaro C, Di Mauro P et al. Efficacy of the DigniCap System in preventing chemotherapyinduced alopecia in breast cancer patients is not related to patient characteristics or side effects of the device. Int $\mathrm{J}$ Nurs Pract 2020 Sep 22;e12888.

16. Kinoshita T, Nakayama T, Fukuma E et al. Efficacy of Scalp Cooling in Preventing and Recovering From Chemotherapy-Induced Alopecia in Breast Cancer Patients: The HOPE Study. Front Oncol 2019;9:733.

17. Marks DH, Okhovat J-P, Hagigeorges D, et al. The effect of scalp cooling on ClA-related quality of life in breast cancer patients: a systematic review. Breast Cancer Res Treat 2019;175(2):267-276.

18. Van Den Hurk CJ, Mols F, Vingerhoets AJ, Breed WP. Impact ofalopecia and scalp cooling on the well-being of breast cancerpatients. Psychooncology 2010;19:701-709.

19. Van den Hurk CJ, Peerbooms M, van de Poll-Franse LV, NortierJW, Coebergh JW, Breed WP. Scalp cooling for hair preser-vation and associated characteristics in 1411 chemotherapy patients - results of the Dutch Scalp Cooling Registry. ActaOncol 2012;51:497-504.

20. Rugo HS, Melin SA, Voigt J. Scalp cooling with adjuvant/neoadjuvant chemotherapy for breast cancer and the risk of scalp metastases: a systematic review and meta-analysis. Breast Cancer Res Treat 2017;163(2):199-205. 